\title{
Comparison of benefit-risk preferences of patients and physicians regarding cyclooxygenase- 2 inhibitors using discrete choice experiments
}

This article was published in the following Dove Press journal:

Patient Preference and Adherence

26 April 2016

Number of times this article has been viewed

\author{
Ji-Hye Byun' \\ Sun-Hong Kwon' \\ Ji-Eun Lee' \\ Ji-Eun Cheon' \\ Eun-Jin Jang ${ }^{2}$ \\ Eui-Kyung Lee'
}

'School of Pharmacy, Sungkyunkwan University, Suwon, Gyeonggi-do, ${ }^{2}$ Information Statistics, Andong National University, Andong, Gyeongsangbuk-do, South Korea
Correspondence: Eui-Kyung Lee School of Pharmacy, Sungkyunkwan University, 300 Cheonchoen-dong, Jangan-gu, Suwon, Gyeonggi-do 440-746, South Korea

Tel +823 3 2907786

Fax +82312994379

Email ekyung@skku.edu
Purpose: To elucidate and compare benefit-risk preferences among Korean patients and physicians concerning cyclooxygenase-2 (Cox-2) inhibitor treatments for arthritis.

Materials and methods: Subjects included 100 patients with arthritis and 60 board-certified orthopedic surgeon physicians in South Korea. Through a systematic review of the literature, beneficial attributes of using Cox-2 inhibitors were defined as a decrease in the Western Ontario and McMaster Universities Arthritis Index for pain score and improvement in physical function. Likewise, risk attributes included upper gastrointestinal (GI) complications and cardiovascular (CV) adverse events. Discrete choice experiments were used to determine preferences for these four attributes among Korean patients and physicians. Relative importance and maximum acceptable risk for improving beneficial attributes were assessed by analyzing the results of the discrete choice experiment by using a conditional logit model.

Results: Patients ranked the relative importance of benefit-risk attributes as follows: pain reduction (35.2\%); physical function improvement (30.0\%); fewer CV adverse events $(21.5 \%)$; fewer GI complications (13.4\%). The physicians' ranking for the same attributes was as follows: fewer CV (33.5\%); pain reduction (32.4\%); fewer GI complications (18.1\%); physical function improvement (16.0\%). Patients were more willing than physicians to accept risks when pain improved from $20 \%$ or $45 \%$ to $55 \%$ and physical function improved from $15 \%$ or $35 \%$ to $45 \%$.

Conclusion: We confirmed that patients and physicians had different benefit-risk preferences regarding Cox-2 inhibitors. Patients with arthritis prioritized the benefits of Cox-2 inhibitors over the risks; moreover, in comparison with the physicians, arthritis patients were more willing to accept the trade-off between benefits and risks to achieve the best treatment level. To reduce the preference gap and achieve treatment goals, physicians must better understand their patients' preferences.

Keywords: discrete choice experiment, Cox-2 inhibitors, willingness to accept, patient and physicians preference, arthritis

\section{Introduction}

Among Korean patients aged 18 years and over, $14.6 \%$ have arthritis. ${ }^{1}$ Arthritis affects patients' quality of life by causing pain and impairing physical joint function. Korean patients generally take selective cyclooxygenase-2 (Cox-2) inhibitors in nonsteroidal anti-inflammatory drugs (NSAIDs) as a palliative therapy to reduce arthritis symptoms. While the beneficial effects of NSAIDs, such as pain reduction and functional improvement, are well established, they cause progressive gastrointestinal (GI) adverse events including nausea, vomiting, dyspepsia, and abdominal pain. ${ }^{2-5}$ Cox -2 inhibitors 
increase the risk of thrombotic cardiovascular (CV) events, such as myocardial infarctions and stroke. ${ }^{6,7}$ Rofecoxib was representatively withdrawn because of reporting adverse event after approval. ${ }^{8}$

Typically, regulatory agencies approve medicines that have benefits that outweigh their risks; however, the benefit-risk balance can be altered after approval owing to additional information regarding efficacy and adverse events. In this respect, major regulatory agencies, such as the European Medicines Agency and the Food and Drug Administration (FDA), and research institutes, such as the Innovative Medicines Initiative, are interested in developing quantitative methods for evaluating the benefit-risk balance and integrating benefit-risk trade-offs in all decision making, even that of patients. ${ }^{9-11}$ In addition, the importance of patients' preference has been increasingly emphasized in recent years, and, this is the reason why differences between two groups can limit the efficacy of drug treatments. ${ }^{12,13}$ To increase the likelihood of successful treatment outcomes, physicians should understand and respect patient preferences. Nevertheless, there was little evidence of comparison with the preference between two groups.

This study was aimed at quantifying the benefit-risk preferences of Korean patients and physicians using discrete choice experiments (DCEs), the results of which were used to assess the relative importance of benefits and maximum acceptable risk (MAR) during the decision to treat arthritis with Cox-2 inhibitors.

\section{Materials and methods DCE development}

DCEs have been utilized to quantitatively identify benefitrisk preference-weights for Cox-2 inhibitors. ${ }^{14-18}$ DCEs afford systematic evaluation of preferences in diverse treatments using trade-offs between attributes. Preferences are elucidated by having respondents repeatedly choose their preferred hypothetical option.

\section{Defining attributes and levels through systematic review}

We included the best-selling celecoxib, along with etoricoxib, which sells differently by country, and rofecoxib, which has been withdrawn. This was because the objective of this study was to elicit preferences for the benefits and risks of Cox-2 inhibitors, and we intended to include the comprehensive preference level from the least preferred to most preferred by selecting three Cox-2 inhibitors.

Developing a DCE required a systematic review to define the attributes and levels for hypothetical treatment outcomes.
Systematic literature reviews to collect data regarding the use of each selected Cox-2 inhibitor in osteoarthritis (OA) or rheumatoid arthritis patients were performed between January 1996 and August 2014. Data were collected from publicly available FDA drug documents, ${ }^{19-24}$ and published clinical trials and observational studies ${ }^{25-43}$ accessed through MEDLINE, and the Cochrane Central Register of Controlled Trials. According to predefined inclusion criteria, six FDA drug documents were selected, while 38 clinical trials from selected reports were included. Our inclusion criteria for data were defined as follows: 1) adult OA or rheumatoid arthritis patients, 2) subjects reported a change in index scores for main symptom relief while taking one of the three selected Cox-2 inhibitors over 4 weeks, 3) subjects reported serious adverse events, 4) reports from randomized clinical trials or observational studies, and 5) evidence reported in English or Korean.

During the literature review, four attributes were extracted from FDA drug documents and selected papers. Two primary beneficial end points from the literature were defined: 1) reduction rate in the Western Ontario and McMaster Universities Arthritis Index (WOMAC) pain score from baseline (PAI) and 2) a reduction rate in WOMAC physical function scale from baseline (FUN). The WOMAC scores are commonly utilized to evaluate the efficacy of treatment for arthritis and it allows for the comparison of the efficacy of Cox-2 inhibitor treatment for arthritis patients. The WOMAC consists of three parts allowing assessment of pain, stiffness, and joint function. In the WOMAC scores, improvement in individual subjects was defined as a decrease in the pain and physical function scores. ${ }^{44}$ However, in this study, subjects did not directly undergo assessment of their WOMAC scores and the WOMAC pain and physical functional scores for three Cox-2 inhibitor drugs were extracted through a systematic review. Risk attributes were defined from serious adverse events reported frequently throughout the literature, including 1) upper GI complications and 2) CV adverse events.

To define response levels for the selected attributes in the questionnaires for two hypothetical Cox-2 inhibitors, we performed mixed treatment comparisons (MTC) for rofecoxib, celecoxib, and etoricoxib. The MTC were necessary because of inadequate direct comparisons between the treatment outcomes of the three selected Cox-2 inhibitors in the literature. The levels used in quantifying the attributes were based on the median results from the MTC; this analysis was conducted using WinBUGS 1.4.3 statistical software (MRC Biostatistics Unit, Cambridge, England) to estimate the values for each Cox-2 inhibitor attribute. The random-effects MTC model was 
Table I Treatment attributes and levels of cyclooxygenase-2 inhibitors

\begin{tabular}{|c|c|c|c|c|}
\hline Attributes & Definition & Level (\%) & Variable name & Coefficients \\
\hline \multicolumn{5}{|l|}{ Benefits } \\
\hline \multirow[t]{3}{*}{ Reduction in pain } & Reduction rate in the WOMAC pain & 20 & $\mathrm{PAI}_{1}$ & $\beta_{\text {PAll }}$ \\
\hline & scale from baseline & 45 & $\mathrm{PAI}_{2}$ & $\beta_{\mathrm{PA} 12}$ \\
\hline & & 55 & $\mathrm{PAl}_{3}$ & $\beta_{\mathrm{PA} \mid 3}$ \\
\hline Improvement of physical & Reduction rate in WOMAC physical & 15 & $\mathrm{FUN}_{1}$ & $\beta_{\mathrm{FUNI}}$ \\
\hline \multirow[t]{2}{*}{ function } & function scale from baseline & 35 & $\mathrm{FUN}_{2}$ & $\beta_{\mathrm{FUN} 2}$ \\
\hline & & 45 & $\mathrm{FUN}_{3}$ & $\beta_{\mathrm{FUN} 3}$ \\
\hline \multicolumn{5}{|l|}{ Risks } \\
\hline Upper Gl & Chance of upper $\mathrm{Gl}$ complication & I (I of I00 patients) & $\mathrm{Gl}_{1}$ & $\beta_{\mathrm{G} \|}$ \\
\hline \multirow[t]{2}{*}{ complications } & (gastric ulcer, Gl bleeding, & 3 (3 of I00 patients) & $\mathrm{GI}_{2}$ & $\beta_{\mathrm{G} 12}$ \\
\hline & or perforation) & 5 (5 of 100 patients) & $\mathrm{Gl}_{3}$ & $\beta_{\mathrm{G} 13}$ \\
\hline \multirow[t]{3}{*}{$\mathrm{CV}$ adverse events } & Chance of $\mathrm{CV}$ adverse events & 0.4 (4 of I,000 patients) & $\mathrm{CV}_{1}$ & $\beta_{\mathrm{cvl}}$ \\
\hline & (angina, myocardial infarction, & 1.2 (I2 of I,000 patients) & $\mathrm{CV}_{2}$ & $\beta_{\mathrm{cv} 2}$ \\
\hline & congestive heart failure, stroke) & 4 (40 of I,000 patients) & $\mathrm{CV}_{3}$ & $\beta_{\mathrm{cv} 3}$ \\
\hline
\end{tabular}

Note: ${ }_{1,2}$ and ${ }_{3}$ indicate the three evaluation levels.

Abbreviations: CV, cardiovascular; FUN, reduction rate in WOMAC physical function scale from baseline; GI, gastrointestinal; PAI, reduction rate in the WOMAC pain scale from baseline; WOMAC, Western Ontario and McMaster Universities Arthritis Index.

used because there was heterogeneity among the rofecoxib, celecoxib, and etoricoxib studies. ${ }^{45}$ The four selected attributes, each with three evaluation levels, are shown in Table 1.

\section{Designing the questionnaires for survey using DCE approach}

The hypothetical options were designed to satisfy orthogonality, minimum overlapping, and equal balance. ${ }^{46}$ The experimental design was optimized using D-efficiency from the SAS software package (version 9.3, SAS Institute Inc., Cary, NC, USA). Eighteen choice-based questionnaire sets were generated and checked for internal consistency and rationality. In each questionnaire, all respondents had to select their preferred Cox-2 treatment between two hypothetical alternatives (Figure 1).

\section{Survey subjects}

One hundred Korean patients and 60 Korean physicians were recruited from a Hankook Research (Gangnam, Seoul, South Korea) panel. Patients were selected according to the following criteria: 1) have a diagnosis of OA or rheumatoid arthritis in panel clinics, 2) set a quote similarly reflecting the sex and age of the target patients, 3) take oral Cox-2 inhibitors or other NSAIDs, and 4) subjects sign the participation consent form in the surveys. Interviewers confirmed whether patients had a diagnosis from their doctors. Physicians in the panel were required to be board-certified orthopedic surgeons in South Korea (Figure 2). All respondents were interviewed by an educated interviewee to reduce bias. The study and survey questionnaires used, were approved by the Sungkyunkwan University Institutional Review Board (Approval No SKKU-2014-07-002-002).

\section{Data analysis}

We compared patients' and physicians' preferences for the benefits and risks of Cox-2 inhibitors across various aspects, including preference weight, perception, relative importance, and MAR.

The preference data for patients and physicians were estimated by conditional logistic regression using SAS 9.3

\begin{tabular}{|c|c|c|c|}
\hline & Attributes & Treatment A & Treatment B \\
\hline \multirow{2}{*}{ Benefits } & Reduction pain & $45 \%$ & $20 \%$ \\
\hline & $\begin{array}{l}\text { Improvement of physical } \\
\text { function }\end{array}$ & $35 \%$ & $45 \%$ \\
\hline \multirow{2}{*}{ Risks } & $\begin{array}{l}\text { Upper gastrointestinal } \\
\text { complication }\end{array}$ & $3 \%$ & $1 \%$ \\
\hline & $\begin{array}{c}\text { Cardiovascular adverse } \\
\text { events }\end{array}$ & $1.2 \%$ & $0 \%$ \\
\hline & vould you prefer to rece & & \\
\hline
\end{tabular}

Figure I Example of a choice set. 


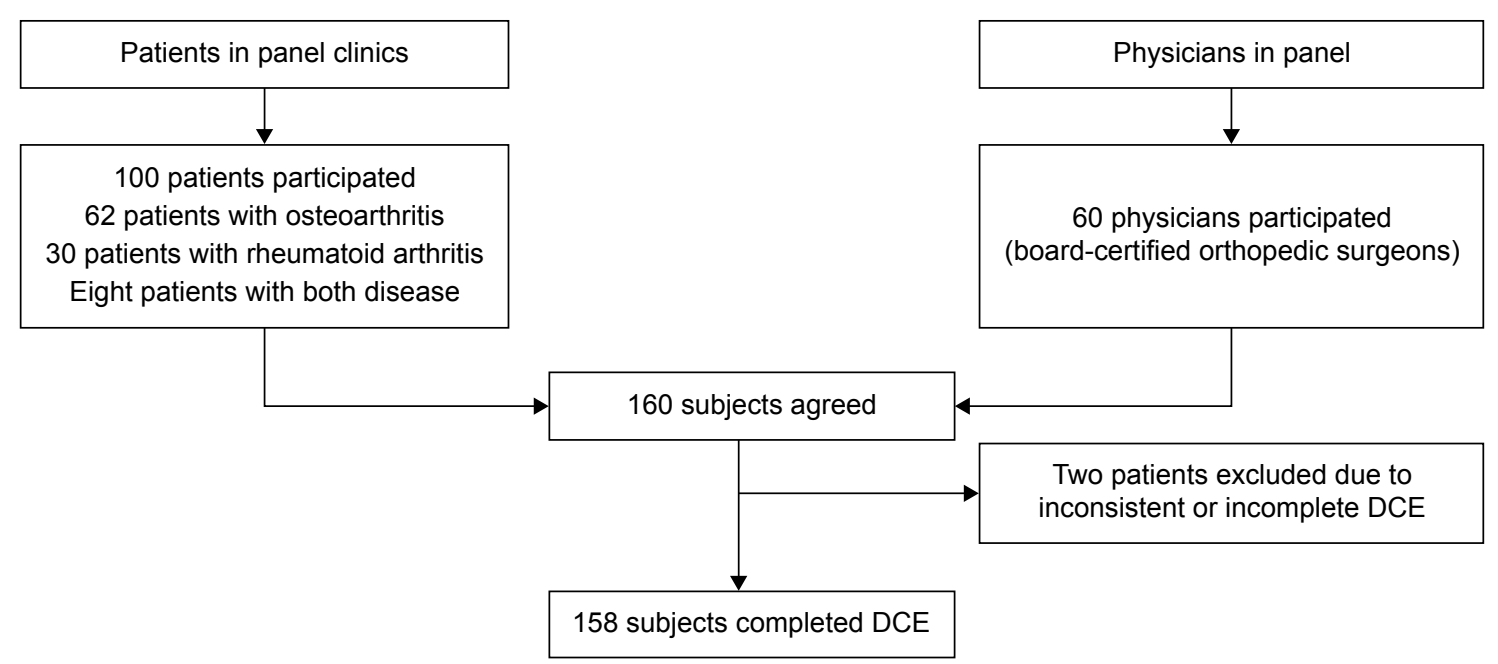

Figure 2 Flowchart for subject selection process. Abbreviation: DCE, discrete choice experiment.

software; respondents who did not select the dominant option were excluded. Data were categorized by an effects-coded model based on previously published guidelines. ${ }^{47}$ The coefficients in this model were estimated for each group; these coefficients represented the preference for each level within each attribute of each group. A positive coefficient for an attribute level indicates a preference for that level over other levels within that attribute. Conversely, a negative coefficient for an attribute level indicates a reduced preference for that level over other levels within that attribute. Accordingly, a higher coefficient means a more preferred level in each attribute. A statistically significant coefficient indicates that the attribute level influences the respondents' treatment decisions.

The equation of the conditional logit model was as follows:

$U$ (binary choice)

$$
\begin{aligned}
= & P A T \times\left(\beta_{\mathrm{PAI} 1} \times \mathrm{PAI}_{1}+\beta_{\mathrm{PAI} 2} \times \mathrm{PAI}_{2}+\beta_{\mathrm{PAI} 3} \times \mathrm{PAI}_{3}\right. \\
& +\beta_{\mathrm{FUN} 1} \times \mathrm{FUN}_{1}+\beta_{\mathrm{FUN} 2}+\mathrm{FUN}_{2}+\beta_{\mathrm{FUN} 3} \times \mathrm{FUN}_{3} \\
& +\beta_{\mathrm{GI}} \times \mathrm{GI}_{1}+\beta_{\mathrm{GI} 2} \times \mathrm{GI}_{2}+\beta_{\mathrm{G} 13} \times \mathrm{GI}_{3}+\beta_{\mathrm{CV} 1} \times \mathrm{CV}_{1} \\
& \left.+\beta_{\mathrm{CV} 2} \times \mathrm{CV}_{2}+\beta_{\mathrm{CV} 3} \times \mathrm{CV}_{3}\right)+P H Y \times\left(\beta_{\mathrm{PAIl}} \times \mathrm{PAI}_{1}\right. \\
& +\beta_{\mathrm{PAI} 2} \times \mathrm{PAI}_{2}+\beta_{\mathrm{PAI} 3} \times \mathrm{PAI}_{3}+\beta_{\mathrm{FUN} 1} \times \mathrm{FUN}_{1} \\
& +\beta_{\mathrm{FUN} 2} \times \mathrm{FUN}_{2}+\beta_{\mathrm{FUN} 3} \times \mathrm{FUN}_{3}+\beta_{\mathrm{GI} 1} \times \mathrm{GI}_{1} \\
& +\beta_{\mathrm{GI} 2} \times \mathrm{GI}_{2}+\beta_{\mathrm{Gl} 3} \times \mathrm{GI}_{3}+\beta_{\mathrm{CV} 1} \times \mathrm{CV}_{1}+\beta_{\mathrm{CV} 2} \times \mathrm{CV}_{2} \\
& \left.+\beta_{\mathrm{CV} 3} \times \mathrm{CV}_{3}\right)
\end{aligned}
$$

where $U$ is the binary choice for the hypothetical Cox-2 inhibitor pair and $P A T$ and $P H Y$ are dummy variables. $P A T$ was 1 and $P H Y$ was 0 when the subjects were patients. Other variables are shown in Table 1 . The $Z$-test was conducted to compare the coefficient between patients and physicians. This approach is utilized to compare the coefficient in regression which was estimated via maximum likelihood such as a conditional logit model. ${ }^{48}$

Preference weight, indicating the relative degree of preference for that specific level or attribute, was calculated using the coefficient difference between one level and the other level within a specific attribute. It was interpreted as the subjects' perception of the change in benefit-risk attribute level. ${ }^{49} \mathrm{We}$ compared each groups' perceptions of changes in benefit and risk outcomes. If the respondents perceived an equal value for changing levels within a specific attribute, then the preference weight was equal. For example, the respondent had equal perception if the preference weight for improving from the moderate level to the best level within the PAI attribute was equal to 1 and the preference weight for declining from the moderate level to the worst level within the CV attribute was 1.

Furthermore, we estimated relative importance as a subset of the preference weights. The relative importance is the relative preference weight for each attribute over all attributes. The greater the relative importance between the four attributes, the more significant the attribute was for decision making.

Lastly, MAR was estimated using the coefficient ratio from the regression. MAR is interpreted as the highest level of treatment-related risk that respondents were willing to accept. ${ }^{15}$ MAR indicated the trade-off between beneficial attributes (PAI and FUN) and risk attributes (GI and CV). We used Microsoft Excel (Microsoft Corporation, Redmond, WA, USA) and 1,000 bootstrap values to determine the MAR with $95 \%$ confidence intervals (CIs). 


\section{Results}

Of the 160 interviewees, two were excluded owing to inconsistent or incomplete responses. The remaining 158 respondents consisted of 98 patients and 60 physicians. From this DCE approach, $98.8 \%$ of respondents rationally preferred the dominant Cox-2 inhibitor when informed regarding the trade-offs between the benefit-risk attributes, meaning that patients and physicians gave their attention to the questionnaire. Table 2 summarizes descriptive statistics for the 158 respondents included in the study. The patient respondents had a mean (standard deviation [SD]) age of 55.7 (7.8) years and 69\% were female. In addition, the most commonly diagnosed disease was $\mathrm{OA}$, at $63 \%$, and the most commonly experienced adverse events were GI, at $40 \%$. Forty-five percent of the physicians had more than 10 years of clinical experience and $98 \%$ were male.

\section{Preference weights for benefit-risk of Cox-2 inhibitors using DCE}

The major results of the conditional logit model are shown in Table 3. As expected, patients and physicians preferred Cox-2 inhibitors with improved PAI or FUN outcomes and

Table 2 Respondents' baseline characteristics

\begin{tabular}{ll}
\hline Characteristics & $\begin{array}{l}\text { Analysis included } \\
\text { respondents } \\
(\%)\end{array}$ \\
\hline Patient group & $98(100)$ \\
Age (mean \pm SD) & $55.7 \pm 7.8$ \\
Sex & \\
Male & $30(31)$ \\
Female & $68(69)$ \\
Diagnosed disease & \\
Osteoarthritis & $62(63)$ \\
Rheumatoid arthritis & $29(30)$ \\
Both osteoarthritis and rheumatoid arthritis & $7(7)$ \\
Experienced adverse event related & \\
to treatment during the treatment process & \\
None & $37(38)$ \\
Gastrointestinal disease & $40(41)$ \\
Cardiovascular disease & $14(14)$ \\
More than two diseases & $5(5)$ \\
Others & $2(2)$ \\
Physicians (orthopedists) & $60(100)$ \\
Age (mean \pm SD) & $40.0 \pm 4.9$ \\
Sex & \\
Male & $59(98)$ \\
Female & $1(2)$ \\
Underwent treatment for arthritis & \\
$<5$ years & $9(15)$ \\
$5-10$ years & $24(40)$ \\
$>10$ years & $27(45)$ \\
\hline Not Data & \\
& \\
\hline
\end{tabular}

Note: Data is presented as number (\%) unless otherwise stated. Abbreviation: SD, standard deviation. those with reduced occurrences of GI or CV adverse events, as identified by higher coefficients for improved PAI or FUN and by lower coefficients for more severe adverse events. However, differences in the preference for each level within certain attributes between patients and physicians were statistically significant, as confirmed by the Z-test. The preferred outcome rankings for patients and physicians associated with the benefit-risks of Cox-2 inhibitors were also different. For example, patients valued the $55 \%$ reduction in WOMAC scores for pain as the most preferential outcome (coefficient: 1.1 [standard error; SE: 0.10], $P$-value: $<0.0001$ ). Conversely, physicians ranked the lowest $\mathrm{CV}$ adverse event occurrence $(0.4 \%)$ as the most preferred outcome (coefficient: 1.1 [SE: 0.10], $P$-value: $<0.0001$ ). In subgroup analysis, we did not find a statistically significant difference between patients with rheumatoid arthritis and OA.

We also identified the manner in which patients' and physicians' perceptions changed with benefit levels and risk levels using preference weights. Patients displayed preference weights lower than those of physicians for reducing GI adverse events from 5\% to 3\% (Figure 3). Patients displayed a preference weight for improving pain reduction from 55\% to $45 \%$ ( $\Delta 0.7$ units) and a preference weight for reducing the occurrence of GI adverse events from $5 \%$ to $3 \%$ ( $\Delta 0.8$ units). Physicians displayed a preference weight for reducing GI adverse events from $5 \%$ to $3 \%$ ( $\Delta 1.1$ units) and increasing joint function improvement from $15 \%$ to $35 \%$ ( $\Delta 1.2$ units).

In addition, we compared the relative importance ranking of risks and benefits among patient and physician groups (Figure 4). In the present study, Korean patients placed a high value on beneficial attributes, whereas physicians evaluated $\mathrm{CV}$ adverse events as the most important attribute. Among the patients, the relative importance of the benefit attributes, PAI (35.2\%) and FUN (30.0\%), outweighed that of the risk attributes, CV adverse events (21.4\%) and GI adverse events (13.4\%), while the CV adverse event risk outweighed the benefit attributes among physicians. Physicians considered $\mathrm{CV}$ adverse events (33.5\%) as the most important attribute, followed by PAI (32.4\%), GI adverse events (18.1\%), and FUN (16.0\%).

\section{Risk tolerance (MAR)}

We compared the extent to which patients and physicians were willing to trade the level of risks associated with Cox-2 inhibitors for improving PAI or FUN (Table 4).

There was a difference in the willingness of patients and physicians to accept the maximum risk for each benefit attribute. Patients were more willing than physicians 
Table 3 Preferences of patient group and physician group as estimated by the regression model

\begin{tabular}{|c|c|c|c|c|c|c|c|c|}
\hline \multirow[t]{2}{*}{ Attribute } & & \multicolumn{3}{|c|}{ Patient group $(\mathrm{n}=98)$} & \multicolumn{3}{|c|}{ Physician group $(n=60)$} & \multirow{2}{*}{$\frac{\text { Comparison }}{P \text {-value }}$} \\
\hline & & Coefficient & \multirow[t]{2}{*}{ SE } & \multirow[t]{2}{*}{$P$-value } & \multirow[t]{2}{*}{ Coefficient } & \multirow[t]{2}{*}{ SE } & $P$-value & \\
\hline \multicolumn{5}{|l|}{ PAI } & & & & \\
\hline $20 \%$ & $\beta_{\text {PAII }}$ & 0.6 & 0.12 & $<0.0001$ & -1.5 & 0.16 & $<0.0001$ & 0.6 \\
\hline $45 \%$ & $\beta_{\mathrm{PA} 12}$ & 0.1 & 0.07 & $<0.0001$ & 0.5 & 0.09 & $<0.000$ I & 0.1 \\
\hline $55 \%$ & $\beta_{\mathrm{PA} \mid 3}$ & 0.5 & 0.08 & $<0.0001$ & 1.0 & 0.129 & $<0.0001$ & 0.5 \\
\hline \multicolumn{9}{|l|}{ FUN } \\
\hline $15 \%$ & $\beta_{\mathrm{FUNI}}$ & 0.1 & 0.13 & $<0.0001$ & -0.8 & 0.13 & $<0.0001$ & 0.1 \\
\hline $35 \%$ & $\beta_{\mathrm{FUN} 2}$ & 0.0 & 0.07 & 0.1305 & 0.4 & 0.10 & 0.0001 & 0.0 \\
\hline $45 \%$ & $\beta_{\mathrm{FUN} 3}$ & 0.0 & 0.10 & $<0.0001$ & 0.4 & 0.09 & $<0.0001$ & 0.0 \\
\hline \multicolumn{9}{|l|}{ Gl } \\
\hline $\begin{array}{l}1 \% \\
\text { (I of } 100 \text { patients) }\end{array}$ & $\beta_{\mathrm{GII}}$ & 0.0 & 0.09 & $<0.0001$ & 0.5 & 0.10 & $<0.0001$ & 0.0 \\
\hline $\begin{array}{l}3 \% \\
\text { ( } 3 \text { of } 100 \text { patients) }\end{array}$ & $\beta_{\mathrm{G} 12}$ & 0.5 & 0.06 & 0.0017 & 0.3 & 0.09 & 0.0029 & 0.5 \\
\hline $\begin{array}{l}5 \% \\
\text { (5 of } 100 \text { patients) }\end{array}$ & $\beta_{\mathrm{G} 13}$ & 0.1 & 0.10 & $<0.0001$ & -0.8 & 0.12 & $<0.0001$ & 0.1 \\
\hline \multicolumn{9}{|l|}{$\mathrm{CV}$} \\
\hline $\begin{array}{l}0.4 \% \\
\text { (4 of I,000 patients) }\end{array}$ & $\beta_{\mathrm{cvl}}$ & 0.0 & 0.10 & $<0.0001$ & 1.1 & 0.11 & $<0.0001$ & 0.0 \\
\hline $\begin{array}{l}1.2 \% \\
\text { ( } 12 \text { of } I, 000 \text { patients) }\end{array}$ & $\beta_{\mathrm{cv} 2}$ & 0.6 & 0.07 & 0.0106 & 0.2 & 0.08 & 0.004 & 0.6 \\
\hline $\begin{array}{l}4.0 \% \\
\text { (40 of I,000 patients) }\end{array}$ & $\beta_{\mathrm{cV} 3}$ & 0.0 & 0.06 & $<0.0001$ & -1.3 & 0.10 & $<0.000$ I & 0.0 \\
\hline Model fit statistics & & \multicolumn{2}{|c|}{ Without covariates } & \multicolumn{2}{|c|}{ With covariates } & Without covariates & \multicolumn{2}{|c|}{ With covariates } \\
\hline AIC & & \multicolumn{2}{|c|}{$2,037.85$} & \multicolumn{2}{|l|}{$1,669.75$} & $1,247.67$ & \multicolumn{2}{|c|}{900.83} \\
\hline SC & & \multicolumn{2}{|c|}{$2,037.85$} & \multicolumn{2}{|l|}{$\mathrm{I}, 7 \mid 7.64$} & I,247.67 & \multicolumn{2}{|c|}{944.79} \\
\hline$-2 \log L$ & & \multicolumn{2}{|c|}{$2,037.85$} & \multicolumn{2}{|l|}{$\mathrm{I}, 653.75$} & $\mathrm{I}, 247.67$ & \multicolumn{2}{|c|}{884.83} \\
\hline \multicolumn{2}{|c|}{ Testing global null hypothesis: Beta $=0$} & \multicolumn{2}{|c|}{ Chi-square } & \multicolumn{2}{|c|}{$P$-value $>$ chi-square } & Chi-square & \multicolumn{2}{|c|}{$P$-value $>$ chi-square } \\
\hline \multicolumn{2}{|c|}{ Likelihood ratio } & \multicolumn{2}{|c|}{384.10} & $<0.0001$ & & 362.84 & $<0$ & 001 \\
\hline Score & & 333.69 & & $<0.0001$ & & 314.70 & $<0$ & 001 \\
\hline Wald's equation & & 230.43 & & $<0.0001$ & & 213.30 & $<0$ & 001 \\
\hline
\end{tabular}

Note: ${ }_{1,2}$ and ${ }_{3}$ indicate the three evaluation levels.

Abbreviations: AIC, Akaike information criterion; CV, cardiovascular; FUN, reduction rate in WOMAC physical function scale from baseline; GI, gastrointestinal; PAI, reduction rate in the WOMAC pain scale from baseline; SC, Schwarz criterion; SE, standard error; WOMAC, Western Ontario and McMaster Universities Arthritis Index.

to accept risk upon improvement of pain reduction from $20 \%$ or $45 \%$ to $55 \%$ (the best treatment response level). For example, patients were, respectively, willing to accept a $1.5 \%$ risk for GI adverse events (95\% CI: $1.46-1.48$ ) and a $1.4 \%$ risk for $\mathrm{CV}$ adverse events (1.42-1.44) to improve pain reduction from $45 \%$ to $55 \%$, while physicians were willing to accept a $0.6 \%$ risk for GI adverse events $(0.57-0.59)$ and a $0.6 \%$ risk for $\mathrm{CV}$ adverse events (0.61-0.63) to improve pain reduction from $45 \%$ to $55 \%$. A similar trend was found in the trade-off between risk and improving physical joint function.

However, interestingly, patients and physicians had some trends in common with regard to MARs. First, both groups were willing to accept the maximum GI and CV risk when the pain reduction was increased from a $20 \%$ reduction to a $55 \%$ reduction. Second, both groups were similarly willing to accept the maximum risk for improving both benefit attributes regardless of the risk type (GI or $\mathrm{CV}$ adverse event).

\section{Discussion}

In this paper, using a DCE approach, we directly compared the benefit-risk trade-offs as reported by Korean patients and physicians regarding Cox-2 inhibitors as treatments for arthritis, leading to several meaningful results. Our results demonstrated the preference gap between patients and physicians regarding Cox-2 inhibitors. We found that patients were more concerned with the benefits of Cox-2 inhibitors, such as PAI and FUN, than with the risk of GI and CV adverse events, whereas physicians considered $\mathrm{CV}$ adverse events to be the most important attribute among the four tested attributes. In addition, we found patients were more willing than physicians to accept risk upon improvement of both beneficial attributes from their worst levels or moderate levels to their best treatment response levels. With respect to decreased PAI, patients were more willing by $1.6(=2.1 / 1.3)$ times to face a GI risk and by $1.5(=2.1 / 1.4)$ times to face a $\mathrm{CV}$ risk than physicians. Notably, with respect to improved FUN, patients 

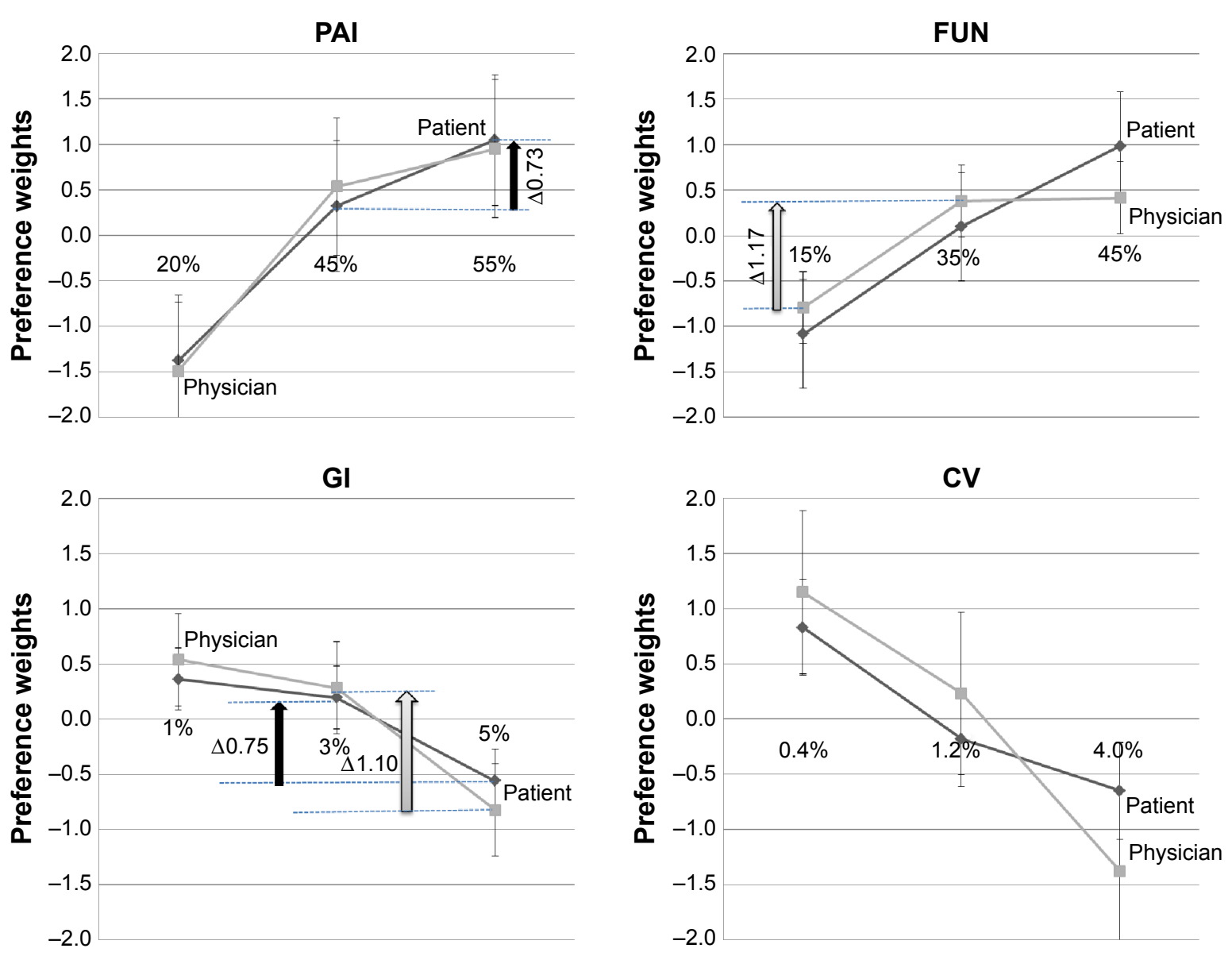

Figure 3 Preference weights for benefit-risk attributes for patient and physician groups.

Notes: The differences between adjacent weights indicate the relative importance of moving from one level of an attribute to an adjacent level of that attributes.

Abbreviations: CV, cardiovascular; FUN, reduction rate in WOMAC physical function scale from baseline; GI, gastrointestinal; PAI, reduction rate in the WOMAC pain scale from baseline; WOMAC, Western Ontario and McMaster Universities Arthritis Index.

were more willing by $3.3(=2.0 / 0.6)$ times to face a GI risk and by $3.2(=1.9 / 0.6)$ times to face a CV risk than physicians, whereas each group ranked the two beneficial attributes (PAI and FUN) and two risk attributes (GI and CV) similarly. With regard to the two benefit attributes, patients and physicians ranked PAI as more important than FUN. With regard

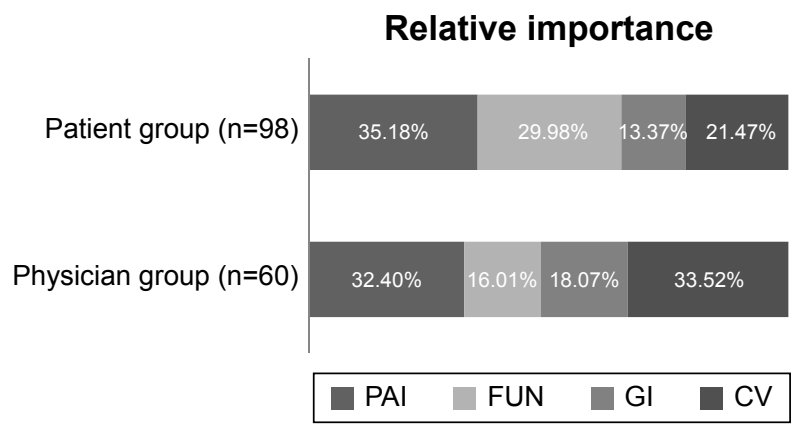

Figure 4 Relative importance of benefit-risk attributes for patient and physician groups. Abbreviations: CV, cardiovascular; FUN, reduction rate in WOMAC physical function scale from baseline; GI, gastrointestinal; PAI, reduction rate in the WOMAC pain scale from baseline; WOMAC, Western Ontario and McMaster Universities Arthritis Index. to the two risk attributes, CV was more important than GI for physicians and patients. These results indicate that lower frequency, more severe risks, such as $\mathrm{CV}$, are considered more important than more frequent, moderate risks when both patients and physicians choose treatment.

Some papers have previously examined benefit-risk preference for NSAIDs including Cox-2 inhibitors. ${ }^{15,16,50,51}$ In these studies, the preference results varied. With respect to examining the preference of physicians, one study showed that risk attributes, such as heart attack risk and stroke risk, outweighed beneficial attributes, such as ambulatory pain and resting pain. ${ }^{15}$ In the present study, Korean physicians displayed preferences similar to those found in previous studies of physicians' preferences. On the other hand, with respect to elucidating the preference of patients, one study demonstrated that patients reported GI risks to be the most important attribute, ${ }^{50}$ while other studies reported that patients evaluated improvement in physical mobility as the most important benefit attribute, ${ }^{51}$ but Korean patients evaluated PAI to be the most important attribute. 
Table 4 MAR for patient and physician groups

\begin{tabular}{|c|c|c|c|c|}
\hline \multirow[t]{2}{*}{ Improvement in benefit } & \multicolumn{2}{|l|}{ Patient group $(n=98)$} & \multicolumn{2}{|l|}{ Physician group $(n=60)$} \\
\hline & GI (mean, [95\% CI]) & CV (mean, [95\% CI]) & GI (mean, [95\% Cl]) & CV (mean, [95\% Cl]) \\
\hline \multicolumn{5}{|l|}{ PAI } \\
\hline $20 \%-45 \%$ & $0.7(0.65-0.67)$ & $0.6(0.63-0.65)$ & $0.8(0.74-0.76)$ & $0.8(0.79-0.8 \mathrm{I})$ \\
\hline $45 \%-55 \%$ & $1.5(1.46-1.48)$ & I.4 (I.42-I.44) & $0.6(0.57-0.59)$ & $0.6(0.6 \mathrm{I}-0.63)$ \\
\hline $20 \%-55 \%$ & $2.1(2.11-2.15)$ & 2.1 (2.05-2.09) & $1.3(1.32-1.34)$ & I.4 (I.4I-I.45) \\
\hline \multicolumn{5}{|l|}{ FUN } \\
\hline $15 \%-35 \%$ & $0.2(0.20-0.20)$ & $0.2(0.20-0.20)$ & $0.5(0.52-0.54)$ & $0.6(0.56-0.58)$ \\
\hline $35 \%-45 \%$ & I.8 (I.79-I.8I) & $1.7(1.72-1.76)$ & $0.1(0.04-0.06)$ & $0.1(0.05-0.07)$ \\
\hline $15 \%-45 \%$ & $2.0(1.99-2.01)$ & $1.9(1.92-1.96)$ & $0.6(0.57-0.59)$ & $0.6(0.6 \mathrm{I}-0.63)$ \\
\hline
\end{tabular}

Abbreviations: $\mathrm{Cl}$, confidence interval; $\mathrm{CV}$, cardiovascular; FUN, reduction rate in WOMAC physical function scale from baseline; GI, gastrointestinal; $\mathrm{MAR}$, maximum acceptable risk; PAI, reduction rate in the WOMAC pain scale from baseline; WOMAC, Western Ontario and McMaster Universities Arthritis Index.

\section{Strengths and limitations}

This comparison study of patients and physicians has a few strengths. This is the first study that quantitatively evaluates the preference for Cox-2 inhibitors and directly compares the preferences between patients and physicians using the DCE method. Furthermore, we defined the essential attributes based on a comprehensive literature review including approval documents opened by the FDA, observational studies, and various clinical trials. Finally, we confirmed that our results were robust while we demonstrated that the measured preferences were statistically significantly different for each level within a given attribute.

However, this study has several limitations. Preferences will manifest differently in the real-world because DCE measures the stated preference, not decision makers' actual choice. ${ }^{52,53}$ Therefore, we need to pay attention to the preference gap between our results and those of the clinical setting. Subsequently, we missed the unobserved systematic interaction and heterogeneous preferences by applying a conditional logit model. This is because the conditional logit model assumes the utility of all subjects is equal..$^{54,55} \mathrm{In}$ spite of this limitation, the conditional logit model is commonly utilized because it efficiently and easily estimates utility. Finally, our study included only orthopedic surgeons among the physicians. Even though a previous study showed no significant differences in the preferences between general physicians and specialists in areas including orthopedics and rheumatology when determining which NSAIDs to prescribe for patients with $\mathrm{OA},{ }^{15}$ we should consider the possibility of a preference gap among general physicians, rheumatology specialists, and orthopedic surgeons in the clinical setting.

\section{Conclusion}

Our study suggests that physicians should more fully consider the benefits to patients' quality of life and should understand that differences in perception between patients and physicians may cause miscommunication when decisions are made regarding patient treatment, in agreement with a previous study. Therefore, to reduce the preference gap between patients and physicians, we suggest that patients should be educated regarding the risks related to treatment with Cox-2 inhibitors; additionally, physicians should set a high value on the benefits to patients' quality of life. Such efforts to reduce the preference gap will help patients with arthritis and physicians successfully reach treatment goals using Cox-2 inhibitors.

\section{Acknowledgment}

This research was supported by a grant (14182mfds536) from Ministry of Food and Drug Safety in 2014.

\section{Disclosure}

The authors report no conflicts of interest in this work.

\section{References}

1. Hur NW, Choi CB, Uhm WS, Bae SC. The prevalence and trend of arthritis in Korea: results from Korea National health and nutrition examination surveys. J Korean Rheum Assoc. 2008;15:11-26.

2. McAlindon TE, Bannuru RR, Sullivan MC, et al. OARSI guidelines for the non-surgical management of knee osteoarthritis. Osteoarthritis Cartilage. 2014;22:363-388.

3. Dai C, Stafford RS, Alexander GC. National trends in cyclooxygenase-2 inhibitor use since market release: nonselective diffusion of a selectively cost-effective innovation. Arch Intern Med. 2005;165:171-177.

4. Garcia Rodríguez LA, Hernández-Díaz S. The risk of upper gastrointestinal complications associated with nonsteroidal anti-inflammatory drugs, glucocorticoids, acetaminophen, and combinations of these agents. Arthritis Res. 2001;3:98-101.

5. Castellsague J, Riera-Guardia N, Calingaert B, et al; Safety of NonSteroidal Anti-Inflammatory Drugs (SOS) Project. Individual NSAIDs and upper gastrointestinal complications: a systematic review and metaanalysis of observational studies (the SOS project). Drug Saf. 2012; 35(12):1127-1146.

6. Jüni P, Nartey L, Reichenbach S, Sterchi R, Dieppe PA, Egger M. Risk of cardiovascular events and rofecoxib: cumulative meta-analysis. Lancet. 2004;364:2021-2029.

7. Mukherjee D, Nissen SE, Topol EJ. Risk of cardiovascular events associated with selective COX-2 inhibitors. JAMA. 2001;286:954-959. 
8. U.S. Food and Drug Administration (FDA). FDA Public Health Advisory: Safety of Vioxx. Available from: http://www.fda.gov/Drugs/DrugSafety/ PostmarketDrugSafetyInformationforPatientsandProviders/ucm106274. htm. Accessed July 14, 2015.

9. European Medicines Agency (EAM) Working Group 2. Benefit-risk methodology project Work package 2 report: Applicability of current tools and processes for regulatory benefit-risk assessment. Available from: http://www.ema.europa.eu/docs/en_GB/document_library/ Report/2010/10/WC500097750.pdf. Accessed July 2, 2015.

10. Frey P. Benefit-risk considerations in CDER: Development of a qualitative framework [Internet]. Center for Drug Evaluation and Research (FDA) DIA Meeting 2012. Available from: http://www.fda.gov/downloads/AboutFDA/CentersOffices/OfficeofMedicalProductsandTobacco/ CDER/UCM317788.pdf. Accessed July 2, 2015.

11. Mt-Isa S, Wang N, Hallgreen CE, et al. Review of methodologies for benefit and risk assessment of medication. IMI-PROTECT: London, 2013. Report No: 1. Available from: http://www.imi-protect.eu/ documents/ShahruletalReviewofmethodologiesforbenefitandriskassessmentofmedicationMay2013.pdf. Accessed July 2, 2015.

12. Haynes RB, Devereaux PJ, Guyatt GH. Physicians' and patients' choices in evidence based practice. BMJ. 2002;324:1350.

13. Fiebig DG, Haas M, Hossain I, Street DJ, Viney R. Decisions about Pap tests: what influences women and providers? Soc Sci Med. 2009;68: 1766-1774.

14. Bryan S, Dolan P. Discrete choice experiments in health economics. For better or for worse? Eur J Health Econ. 2004;5(3):199-202.

15. Arden NK, Hauber AB, Mohamed AF, et al. How do physicians weigh benefits and risks associated with treatments in patients with osteoarthritis in the United Kingdom? J Rheumatol. 2012;39:1056-1063.

16. Hauber AB, Arden NK, Mohamed AF, et al. A discrete-choice experiment of United Kingdom patients' willingness to risk adverse events for improved function and pain control in osteoarthritis. Osteoarthritis Cartilage. 2013;21:289-297.

17. Augustovski F, Beratarrechea A, Irazola V, et al. Patient preferences for biologic agents in rheumatoid arthritis: a discrete-choice experiment. Value Health. 2013;16:385-393. doi:10.1016/j.jval.2012.11.007.

18. Fraenkel L, Suter L, Cunningham CE, Hawker G. Understanding preferences for disease-modifying drugs in osteoarthritis. Arthritis Care Res (Hoboken). 2014;66:1186-1192.

19. Merk, FDA Arthritis Drug Advisory Committee Meeting, Briefing Document (Background Package) - Arcoxib (Etoricoxib), Protocol 007(2002) (September 9, 2015). Available from: http://www.fda.gov/ ohrms/dockets/ac/07/briefing/2007-4290b1-01-FDA.pdf. Accessed September 9, 2015.

20. Merck, FDA Arthritis Drug Advisory Committee Meeting, Briefing Document (Background Package) - Arcoxia (Etoricoxib), Protocol 018(2007) (September 9, 2015). Available from: http://www.fda.gov/ ohrms/dockets/ac/07/briefing/2007-4290b1-01-FDA.pdf

21. Villalba ML, Medical Review P3 - Rofecoxib, Protocol 029(1999) (September 9, 2015). Available from: http://www.accessdata.fda.gov/ drugsatfda_docs/nda/99/021042_52_vioxx_medr_P3.pdf

22. Merck, FDA Arthritis Drug Advisory Committee Meeting, Briefing Document (Background Package) - Arcoxia (Etoricoxib), Protocol 071(2005) (September 9, 2015). Available from: http://www.fda.gov/ ohrms/dockets/ac/07/briefing/2007-4290b1-02-Merck.pdf

23. Merck, FDA Arthritis Drug Advisory Committee Meeting, Briefing Document (Background Package) - Arcoxia (Etoricoxib), Protocol 076(2007) (September 9, 2015). Available from: http://www.fda.gov/ ohrms/dockets/ac/07/briefing/2007-4290b1-02-Merck.pdf

24. Merck, FDA Arthritis Drug Advisory Committee Meeting, Briefing Document (Background Package) - Arcoxia (Etoricoxib), Protocol 077(2007) (September 9, 2015). Available from: http://www.fda.gov/ ohrms/dockets/ac/07/briefing/2007-4290b1-02-Merck.pdf

25. Bingham CO, 3rd, Sebba AI, Rubin BR, et al. Efficacy and safety of etoricoxib $30 \mathrm{mg}$ and celecoxib $200 \mathrm{mg}$ in the treatment of osteoarthritis in two identically designed, randomized, placebo-controlled, non-inferiority studies. Rheumatology (Oxford). 2007;46(3): 496-507.
26. Birbara C, Ruoff G, Sheldon E, et al. Efficacy and safety of rofecoxib $12.5 \mathrm{mg}$ and celecoxib $200 \mathrm{mg}$ in two similarly designed osteoarthritis studies. Curr Med Res Opin. 2006;22(1):199-210.

27. Cannon GW, Caldwell JR, Holt P, et al. Rofecoxib, a specific inhibitor of cyclooxygenase 2, with clinical efficacy comparable with that of diclofenac sodium: results of a one-year, randomized, clinical trial in patients with osteoarthritis of the knee and hip. Rofecoxib Phase III Protocol 035 Study Group. Arthritis Rheum. 2000;43(5):978-987.

28. Cheung R, Cheng TT, Dong Y, et al. Incidence of gastroduodenal ulcers during treatment with celecoxib or diclofenac: pooled results from three 12-week trials in Chinese patients with osteoarthritis or rheumatoid arthritis. Int J Rheum Dis. 2010;13(2):151-157.

29. Collantes E, Curtis SP, Lee KW, et al. A multinational randomized, controlled, clinical trial of etoricoxib in the treatment of rheumatoid arthritis [ISRCTN25142273]. BMC Fam Pract. 2002;3:10.

30. Curtis SP, Bockow B, Fisher C, et al. Etoricoxib in the treatment of osteoarthritis over 52-weeks: a double-blind, active-comparator controlled trial [NCT00242489]. BMC Musculoskelet Disord. 2005;6:58.

31. Dahlberg LE, Holme I, Høye K, Ringertz B. A randomized, multicentre, double-blind, parallel-group study to assess the adverse event-related discontinuation rate with celecoxib and diclofenac in elderly patients with osteoarthritis. Scand J Rheumatol. 2009;38(2):133-143.

32. Day R, Morrison B, Luza A, et al. A randomized trial of the efficacy and tolerability of the Cox-2 inhibitor rofecoxib vs ibuprofen in patients with osteoarthritis. Rofe-coxib/Ibuprofen Comparator Study Group. Arch Intern Med. 2000;160(12):1781-1787.

33. Emery P, Zeidler H, Kvien TK, et al. Celecoxib versus diclofenac in long-term management of rheumatoid arthritis: randomised doubleblind comparison. Lancet. 1999;354(9196):2106-2111.

34. Essex MN, Behar R, O'Connell MA, Brown PB. Efficacy and tolerability of celecoxib and naproxen versus placebo in Hispanic patients with knee osteoarthritis. Int J Gen Med. 2014;7:227-235.

35. Geba GP, Weaver AL, Polis AB, Dixon ME, Schnitzer TJ; Vioxx, Acetaminophen, Celecoxib Trial (VACT) Group. Efficacy of rofecoxib, celecoxib, and acetaminophen in osteoarthritis of the knee: a randomized trial. JAMA. 2002;287(1):64-71.

36. Gottesdiener K, Schnitzer T, Fisher C, et al; Protocol 007 Study Group. Results of a randomized, dose-ranging trial of etoricoxib in patients with osteoarthritis. Rheumatology (Oxford). 2002;41(9): 1052-1061.

37. Hawkey C, Laine L, Simon T, et al. Comparison of the effect of rofecoxib(a cyclooxygenase 2 inhibitor), ibuprofen, and placebo on the gastroduodenal mucosa of patients with osteoarthritis: a randomized, double-blind, placebo-controlled trial. The Rofecoxib Osteoarthritis Endoscopy Multinational Study Group. Arthritis Rheum. 2000;43(2): 370-377.

38. Laine L, Harper S, Simon T, et al. A randomized trial comparing the effect of rofecoxib, a cyclooxygenase 2-specific inhibitor, with that of ibuprofen on the gastroduodenal mucosa of patients with osteoarthritis. Rofecoxib Osteoarthritis Endoscopy Study Group. Gastroenterology. 1999;117(4):776-783.

39. Leung AT, Malmstrom K, Gallacher AE, et al. Efficacy and tolerability profile of etoricoxib in patients with osteoarthritis: A randomized, double-blind, placebo and active-comparator controlled 12-week efficacy trial. Curr Med Res Opin. 2002;18(2):49-58.

40. Matsumoto AK, Melian A, Mandel DR, et al; Etoricoxib Rheumatoid Arthritis Study Group. A randomized, controlled, clinical trial of etoricoxib in the treatment of rheumatoid arthritis. J Rheumatol. 2002;29(8): $1623-1630$

41. Cannon CP, Curtis SP, FitzGerald GA, et al; MEDAL Steering Committee. Cardiovascular outcomes with etoricoxib and diclofenac in patients with osteoarthritis and rheumatoid arthritis in the Multinational Etoricoxib and Diclofenac Arthritis Long-term (MEDAL) programme: a randomised comparison. Lancet. 2006;368(9549):1771-1781.

42. Reginster JY, Malmstrom K, Mehta A, et al. Evaluation of the efficacy and safety of etoricoxib compared with naproxen in two, 138-week randomised studies of patients with osteoarthritis. Ann Rheum Dis. 2007;66(7):945-951. 
43. Simon LS, Weaver AL, Graham DY, et al. Anti-inflammatory and upper gastrointestinal effects of celecoxib in rheumatoid arthritis: a randomized controlled trial. JAMA. 1999;282(20):1921-1928.

44. Bellamy N, Buchanan WW, Goldsmith CH, Campbell J, Stitt LW. Validation study of WOMAC: a health status instrument for measuring clinically important patient relevant outcomes to antirheumatic drug therapy in patients with osteoarthritis of the hip or knee. J Rheumatol. 1988; 15(12):1833-1840.

45. Higgins JP, Thompson SG, Spiegelhalter DJ. A re-evaluation of random-effects meta-analysis. J R Stat Soc Ser A Stat Soc. 2009;172: 137-159.

46. Hedayat AS, Sloane NJA, Stufken J. Orthogonal Arrays: Theory and Applications. New York: Springer Verlag; 1999.

47. Reed Johnson F, Lancsar E, Marshall D, et al. Constructing experimental designs for discrete-choice experiments: report of the ISPOR Conjoint Analysis Experimental Design Good Research Practices Task Force. Value Health. 2013;16:3-13.

48. Morillas C, Feliciano R, Catalina PF, et al. Patients' and physicians' preferences for type 2 diabetes mellitus treatments in Spain and Portugal: a discrete choice experiment. Patient Prefer Adherence. 2015;9: 1443-1458

49. Kauf TL, Mohamed AF, Hauber AB, Fetzer D, Ahmad A. Patients' willingness to accept the risks and benefits of new treatments for chronic hepatitis C virus infection. Patient. 2012;5(4):265-278.
50. Fraenkel L, Bogardus ST Jr, Concato J, Wittink DR. Treatment options in knee osteoarthritis: the patient's perspective. Arch Intern Med. 2004;164: 1299-1304.

51. Ratcliffe J, Buxton M, McGarry T, Sheldon R, Chancellor J. Patients' preferences for characteristics associated with treatments for osteoarthritis. Rheumatology (Oxford). 2004;43:337-345.

52. Viney R, Lancsar E, Louviere J. Discrete choice experiments to measure consumer preference for health and healthcare. Expert Rev Pharmacoecon Outcomes Res. 2002;2(4):89-96.

53. Bridges JFP, Hauber AB, Marshall D, et al. Conjoint analysis applications I health: a checklist of the ISPOR good research practices for conjoint analysis task force. Value Health. 2011;14:403-413.

54. Report of the ISPOR Conjoint Analysis Good Research Practices Task Force. Methods for the Statistical Analysis of Discrete-Choice Experiments: Available from: http://www.ispor.org/TaskForces/ConjointAnalysis-Statistical-Analysis-forum-Milan\%202015.pdf. Accessed February 12, 2016.

55. Clark MD, Determann D, Petrou S, Moro D, de Bekker-Grob EW. Discrete choice experiments in health economics: a review of the literature. Pharmacoeconomics. 2014;32(9):883-902.
Patient Preference and Adherence

\section{Publish your work in this journal}

Patient Preference and Adherence is an international, peer-reviewed, open access journal that focuses on the growing importance of patient preference and adherence throughout the therapeutic continuum. Patient satisfaction, acceptability, quality of life, compliance, persistence and their role in developing new therapeutic modalities and compounds to optimize

\section{Dovepress}

clinical outcomes for existing disease states are major areas of interest for the journal. This journal has been accepted for indexing on PubMed Central. The manuscript management system is completely online and includes a very quick and fair peer-review system, which is all easy to use. Visit http://www. dovepress.com/testimonials.php to read real quotes from published authors. 